\title{
RESEARCH OF INFLUENCE OF THE EARTH'S SUBSIDENCE OUTSIDE THE LOADS CONTOUR ON THE STABILITY OF THE HEIGHT POSITION OF THE POINTS OF LOCAL LEVELING NETWORKS
}

\author{
Myroslav DUTCHYN ${ }^{1}$, Tetyana GRYTSYUK ${ }^{1}$, Iryna BIDA ${ }^{1}$, Lyubov DOROSH ${ }^{1}{ }^{*}$, \\ Rostyslav PYLYPYUK ${ }^{1}$, Mariya NYCHVYD ${ }^{2}$ \\ ${ }^{1}$ Ivano-Frankivsk National Technical University of Oil and Gas, Ivano-Frankivsk, Ukraine \\ ${ }^{2}$ Uzhgorod National University, Uzhgorod, Ukraine
}

Received 07 October 2019; accepted 15 September 2020

\begin{abstract}
The influence of static loadings of the foundations of structures in the process of soil compaction of the base on the subsidence of the soil surface and the bench outside the loaded contour is considered in the work. The studies were performed on models of rectangular massive foundations using the method of equivalent soil layer, based on the theory of compaction of linear-deforming half-space. Using this method, the values of the average sedimentation of the models of rectangular foundations were calculated, taking into account all components of normal stresses and lateral soil extension. On the basis of the method of angular points of the equivalent layer, the values of subsidence of the soil surface outside the contour of the foundation models are calculated, depending on the distance from the contour of the foundation, the value of the average subsidence of the foundation and its parameters. To determine the areas of the soil surface most sensitive to the perception of static loads, lines of equal settlements of the soil surface outside the contour of the foundation models are constructed. On the basis of theoretical studies, probable magnitudes of sedimentation of soil benchmarks are calculated, depending on the subsidence of the soil surface and the distance from the contour of the foundation. The probable minimum distance from the contour of the structure to the places of laying of initial soil benchmarks is established to ensure the required accuracy of geodetic observations. The results of the conducted researches may find application in the design of geodetic observations of the settlements and deformations of the foundations of engineering structures and the choice of the locations of local level points.
\end{abstract}

Keywords: foundation, static loads, soil compaction, equivalent soil layer, active compression zone, soil surface sedimentation, stress zone boundary, sedimentation funnel, soil benchmarks.

\section{Introduction}

Monitoring subsidence and deformation of the foundations of engineering structures and technological equipment occupy a significant place in the modern practice of engineering and geodetic works. Moreover, the volume and complexity of observations, as well as the requirements for the accuracy of their implementation are constantly growing.

When organizing geodetic observations of subsidence and deformation of the foundations of structures at the facility, one of the main tasks, as is known, is the choice of the location and installation of points of local leveling networks.

Properly organized observations begin at the same time as designing. In the same period, a control-point system is created in order to detect the degree of their stability before the erection of the structure.
The experience of observations shows (Ganshin et al., 1981; Bolshakov et al., 1976; Levchuk et al., 1980), that as a result of various reasons, which are quite difficult to take into account, the stability of the height position of the output benchmarks is broken.

In the process of soil compaction, a so-called "sedimentation funnel" (deformation zone) is formed around each structure being erected, the area of distribution of which depends, first of all, on a number of physical and mechanical properties of the soil and the weight of the structure, ie on the magnitude of its subsidence.

Since the boundaries of the distribution of the sedimentation funnel are practically undetermined, the benchmarks are, probably, located outside the mobile zone, may shift along with the rocks in which their anchors are laid.

As the results of observations show (Bolshakov et al., 1976), it is necessary to take into account the influence of

${ }^{*}$ Corresponding author. E-mail: liubov.dorosh@gmail.com 
the deformation zone on the displacement of the benchmark, even if it is laid at a sufficiently deep depth.

Permissible for geodetic practice, the boundaries of these displacements are due to the accuracy of determining the heights of the output benchmarks and should not exceed the limits of this accuracy.

Failure to consider this factor can lead to erroneous conclusions about the stability of the original benchmarks and, as a consequence, to misinterpretation of the results of geodetic observations of sedimentation and deformation of the foundations of engineering structures.

Regular observations of the subsidence and deformation of the foundations of buildings and structures often begin after the completion of major construction work, and therefore the magnitude of the settlements during this period remain virtually unknown. Therefore, it is impossible to correctly judge the magnitude of the absolute subsidence of the structure and the possible reasons for the violation of its normal operation.

In the construction of industrial and civil sites, there are cases where for technical or organizational reasons there is no possibility of laying special depth or fundamental benchmarks. However, prior to the commencement of construction work, it is necessary to have starting points, the height of which in each cycle of leveling within the set accuracy would be unchanged.

In such uncertain cases, they are often limited to the installation of conventional soil benchmarks.

It is necessary to take into account both the depth of their laying, and the distance from the contour of the foundation of the structure to limit the influence of technogenic factors on the displacement of the benchmark.

Thus, the project of localization of local leveling points for observation of sedimentation and deformation of the foundations of structures should be developed taking into account the probable subsidence of the soil surface and benchmarks due to the change of natural pressure on the soil during the loading of the foundation of the structures.

\section{Analysis of recent studies and publications regarding the solution to this problem}

A number of publications in the scientific and technical literature are devoted to the questions of the prediction of the settlements of the foundations of engineering structures and the soil surface.

To calculate the settlements of massive reinforced concrete flexible foundations in Tishin (1985), a calculation scheme in the form of an elastic deforming half-space with the method of layer summation and the method of equivalent soil layer is proposed. The advantages and disadvantages of the proposed methods are indicated in the paper.

In the article (Rudy et al., 2001) present algorithms and schemes for calculating the settlements of fixed points of a flexible rectangular foundation using the method of angular points of equivalent layer.
Characteristics of the types of foundations and the scheme of arrangement of deformation marks on the foundations of gas-pumping units of compressor stations are given in Dutchyn (2001). It proposes an optimal scheme for determining the average subsidence of the foundation.

The paper (Perovich \& Dutchyn, 1996) proposes a formula for calculating the value of the average subsidence of the soil surface outside the rectangular foundation.

Algorithms for determining the settlements of points of the soil surface, depending on their location relative to the contour of the rectangular foundation, are given in Dutchyn (1999). According to the results of studies it is found that the value of settling of the soil surface, all things being equal, depends on the position of the point relative to the contour of the foundation.

The paper (Dutchyn \& Melnychenko, 2005) proposes a formula for calculating the maximum subsidence of the soil surface outside the rectangular foundation, depending on the distance from the contour of the foundation, the value of the average subsidence of the foundation and its parameters.

The work (Dutchyn, 2005) is devoted to the study of the accuracy and informativeness of determining the settlements of rectangular foundations in the process of soil compaction, depending on the location of observed marks and the relative size of the foundation. It lists a number of layout schemes for benchmarks and calculates the magnitude of their settlements. Recommendations for choosing the best option are also given.

The magnitudes of the relations between the maximum subsidence of the soil surface outside the loaded contour and the average subsidence of the building foundation for a number of rectangular foundation models are given in Dutchyn et al. (2012a).

The paper (Dutchyn et al., 2012b) presents a method of calculating the allowable values of deformation characteristics of the bases of pumping units of compressor stations, taking into account the functional dependences of sediment on the relative size of the foundation and maintaining the optimal spatial position of the axes of the equipment.

The method of calculation of accuracy of determination of settlements of foundations of engineering structures, based on taking into account inequalities of settlements of points located along the longitudinal axis of the foundation, is considered in the paper (Dutchyn et al., 2014a).

The paper (Dutchyn \& Melnychenko, 2002) proposes a method of determining the permissible sedimentation of the bases of the pumping units of compressor stations under the influence of static loads, taking into account the permissible value of the deflection boom of the lower base plate.

Rationale for the possibility of using soil benchmarks for observations of sedimentation of foundations of engineering structures is given in Dutchyn et al. (2014b).

The results of the calculation of the probable settlements of the soil surface and of the bench outside the 
contour of the rectangular foundation models are given in Dutchyn et al. (2016). It is stated that the value of settling of the surface and the benchmarks depends on the relative size of the foundation of the structure.

The given publications do not pay due attention to the questions of the accuracy of geodetic observations of the settlements the foundations of engineering structures, as well as the stability of the height position of local level points caused by man-made influences.

\section{Problem statement}

Determination of the optimum distance from the contour of the foundation of the structure to the places of laying the points of local leveling networks, providing the required accuracy of geodetic observations of settlements and deformations of the foundations of engineering structures.

\section{Statement of the main material}

The service life of any engineering structure with some degree of convention is divided into two periods. In the first, relatively short period, which includes the term of construction of the object, settlements of the soil surface at the base of the foundation (deformation of soil compaction) occur. It is believed that the so-called "primary settlements" of the structures that arise in this way reach their maximum (up to $80-85 \%$ ) (Ganshin et al., 1981). The first period ends with the conditional stabilization of the physical and mechanical properties of the soils, which ensures the relative stability of their surface at the base of the foundations.

In the following period (at the stage of operation of the structure) there are "secondary settlements", which are mainly a manifestation of soil creep.

Prediction of sedimentation of foundations of structures at the stage of compaction of bases is performed, as a rule, using methods based on the solution of problems on the theory of consolidation (Dashko \& Kachan, 1977; Tsytovych, 1983). The reasons for the possible discrepancies with the experimental data are due both to the difficulty of obtaining sufficiently accurate characteristics of many soil properties that underlie the calculations and to the choice of optimal methods and computational schemes (Dutchyn, 1996).

One of the most accepted for practical purposes engineering methods for predicting the settlements of the foundations of structures at the stage of soil compaction is considered the method of equivalent soil layer (Tsytovych, 1983).

For homogeneous at sufficient depth of soil definition of full stabilized subsidence of foundations by this method is a rigorous solution of the theory of compaction of linear-deforming half-space.

Calculation of subsidence of foundations by the method of equivalent layer of soil is carried out taking into account all components of stresses and lateral expansion of soil. In the case of layering, the soil is quasi-homogeneous with an average coefficient of relative compression along the depth of the core.

The amount of sedimentation and the nature of the deformation of the foundation of the engineering structure at the stage of soil compaction of the foundation largely depend on the construction of the foundation, ie on its rigidity.

On the rigidity and nature of the distribution of deformations, the foundations are divided into two main types: rigid and flexible. Indicators of the flexibility (rigidity) of foundations are largely dependent on the stiffness coefficients of the substrate and are determined by known formulas (Tsytovych, 1983; Zurnadzhi \& Nikolaev, 1967).

The subsidence of all points of absolutely rigid foundation at uniformly distributed load will be the same. At uneven compression of soils the bases of the foundation of this type receive a roll without bending the structure.

For evenly loaded flexible foundations of subsidence at different points of the sole of the foundation will be different.

Vertical displacements of the foundations are estimated, as a rule, by the magnitude of their average subsidence, which is one of the main deformation characteristics of the foundation of the structure.

By comparing the subsidence of an absolutely rigid and absolutely flexible foundation, it is possible to identify points whose subsidence (all other things being equal) does not depend on the rigidity of the foundation and correspond to the average subsidence of both the rigid and the flexible foundation.

According to Shutenko et al. (1989), in accordance with Grasshoff's research, the position of the so-called "characteristic points", whose subsidence corresponds to the value of the mean subsidence of the foundation, regardless of its rigidity, is determined by the coordinates: $X= \pm 0.37 b ; \quad Y= \pm 0.37 l$ (Figure 1).

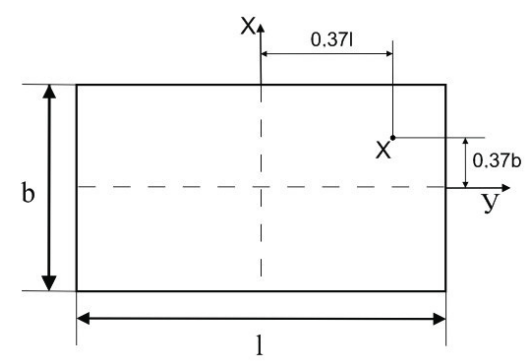

Figure 1. Position of the characteristic point according to Grasshoff

The calculated dependence for calculating additional stresses $G_{Z}$ along the axis passing through the characteristic point of a uniformly loaded rectangular foundation in this method is presented in the form:

$$
G_{Z}=J_{Z} \cdot P \text {, }
$$

where $J_{Z}$ - dimensionless coefficient of variation of additional stresses with depth; $P$ - uniformly distributed load intensity. 
According to other studies (Dutchyn et al., 2007) the position of the characteristic point, calculated by the method of equivalent soil layer, is determined by the following coordinates:

$$
\left\{\begin{array}{l}
X_{x}= \pm\left(0.328+1.372 \cdot 10^{-3} \alpha\right) b \\
Y_{x}= \pm\left(0.328+1.372 \cdot 10^{-3} \alpha\right) l
\end{array}\right.
$$

where $b, l$ - the width and length of the foundation, accordingly, $a$ - aspect ratio of the foundation.

As is known (Tsytovych, 1983; Zurnadzhi \& Nikolaev, 1967), massive reinforced concrete foundations are designed as flexible.

The value of the average stabilized $S_{m}$ foundation sediment in the equivalent soil layer method is determined by the formula (Dashko \& Kachan, 1977; Tsytovych, 1983):

$$
S_{m}=h_{e} \cdot m_{v_{m}} \cdot P_{0},
$$

where $h_{e}$ is the thickness of the equivalent soil layer; $m_{v_{m}}$ - average (reduced) coefficient of relative soil compression; $P_{0}$ - additional soil pressure at the depth of the foundation laying.

The thickness of the equivalent layer of soil is determined by the formula:

$$
h_{e}=A \cdot \omega_{m} \cdot b,
$$

where $A$ is the constant (for this soil) power factor of the equivalent layer; $\omega_{m}$ - the shape factor of the area of the sole and the stiffness of the foundation (when calculating the value of average subsidence); $b$ - the width of the foundation.

The power factor of the equivalent layer is determined by the formula:

$$
A=\frac{\left(1-\mu_{0}\right)^{2}}{\left(1-2 \mu_{0}\right)}
$$

where $\mu_{0}$ is the coefficient of transverse expansion of the soil.

The coefficient value $\omega_{m}$ (function above $\alpha$ ) can be calculated by the formula:

$$
\omega_{m}=1 /\left[\alpha(l / b)^{\tau}\right]
$$

where is the length of the foundation; $b, l-$ the width and length of the foundation, accordingly; $\alpha, \tau$ - coefficients $(\alpha=1.0173 ; \tau=-0.37708)$.

Additional pressure on the soil $P_{0}$ is calculated as (Tsytovych, 1983):

$$
P_{0}=P-\gamma h,
$$

where $P$ is the total pressure on the soil from the weight of the structure (the intensity of the evenly distributed load); $\gamma h$ - natural pressure $(\gamma-$ volumetric weight of the soil $h$ - the depth of the foundation).

Calculations of mean sedimentation values by the method of equivalent soil layer according to the formula (3) were performed on models of rectangular flexible foundations, the parameters of which are shown in Table 1 .

The thickness of the foundation slab, as well as the probable depth of the foundations, was assumed to be $1.8 \mathrm{~m}$.

Table 1. The main parameters of the models of rectangular foundations and their soil bases

\begin{tabular}{|c|c|c|c|c|c|c|c|}
\hline $\begin{array}{c}\text { Mo- } \\
\text { del } \\
\text { num- } \\
\text { bers }\end{array}$ & $\begin{array}{c}1, \\
\mathrm{~m}\end{array}$ & $\begin{array}{c}b, \\
\mathrm{~m}\end{array}$ & $\alpha$ & $\begin{array}{c}F, \\
\mathrm{~m}^{2}\end{array}$ & $\mu_{0}$ & $\begin{array}{c}m_{v_{m}}, \\
\mathrm{~cm}^{2} / \mathrm{H}\end{array}$ & $\begin{array}{c}P_{0}, \\
\mathrm{mPa}\end{array}$ \\
\hline 1 & 12.0 & 4.0 & 3.00 & 48.00 & 0.2 & $4.2410^{-4}$ & 0.0500 \\
\hline 2 & 23.0 & 3.0 & 7.67 & 69.00 & 0.2 & $4.2410^{-4}$ & 0.0500 \\
\hline 3 & 8.0 & 8.0 & 1.00 & 64.00 & 0.2 & $4.2410^{-4}$ & 0.0500 \\
\hline 4 & 20.0 & 4.7 & 4.26 & 94.00 & 0.2 & $4.2410^{-4}$ & 0.0500 \\
\hline 5 & 12.2 & 4.2 & 2.90 & 51.24 & 0.2 & $6.2210^{-4}$ & 0.0603 \\
\hline
\end{tabular}

As can be seen from the above calculations (Table 2), the average subsidence $S_{m}$ of the foundation (for the same values $\left.\mu_{0}, m_{v_{m}}, P_{0}\right)$ depends on the area of the base of the foundation $F$.

The average values $S_{m}$ of sediment models of rectangular foundations, calculated by the formula (3), are shown in Table 2.

Table 2. The main parameters of the models of rectangular foundations and their soil bases

\begin{tabular}{|c|c|c|c|c|c|}
\hline Model numbers & 1 & 2 & 3 & 4 & 5 \\
\hline$S_{m}, \mathrm{~cm}$ & 13.82 & 14.21 & 17.13 & 18.40 & 25.30 \\
\hline
\end{tabular}

Other things being equal $\left(\mu_{0}, m_{v_{m}}, P_{0}, F\right)$, the subsidence $S_{m}$ of the foundation depends on the aspect ratio of the foundation $\alpha$ : the subsidence $S_{m}$ decreases with increasing $a$ (Table 3).

Table 3. Subsidence of foundations depending $a$ on $\mu_{0}=0.2$; $m_{v_{m}}=3.7 \cdot 10^{-4} \mathrm{~cm}^{2} / \mathrm{H} ; \quad P_{0}=2.95 \mathrm{H} / \mathrm{cm}^{2} ; \quad F=68 \mathrm{~m}^{2}$

\begin{tabular}{|c|c|c|c|c|}
\hline$a$ & $l, \mathrm{~m}$ & $b, \mathrm{~m}$ & $h_{e_{m}}, \mathrm{~m}$ & $S_{m}, \mathrm{~mm}$ \\
\hline 1.0 & 8.25 & 8.25 & 8.33 & 9.09 \\
\hline 2.0 & 11.66 & 5.83 & 8.10 & 8.84 \\
\hline 3.0 & 14.28 & 4.76 & 7.76 & 8.47 \\
\hline 4.0 & 16.49 & 4.12 & 7.46 & 8.14 \\
\hline 5.0 & 18.44 & 3.69 & 7.20 & 7.86 \\
\hline 6.0 & 20.20 & 3.37 & 7.04 & 7.68 \\
\hline 7.0 & 21.82 & 3.12 & 6.80 & 7.42 \\
\hline 8.0 & 23.32 & 2.92 & 6.60 & 7.20 \\
\hline 9.0 & 24.74 & 2.75 & 6.44 & 7.03 \\
\hline 10.0 & 26.08 & 2.61 & 6.26 & 6.83 \\
\hline
\end{tabular}

The subsidence of an arbitrary point on the soil surface outside a rectangular foundation can be determined 
using the method of corner points of an equivalent layer (for a spatial problem) using the formula (Dutchyn, 1999; Dutchyn \& Melnychenko, 2005):

$$
S_{r}=\left[h_{e}\right] \cdot m_{v_{m}} \cdot P,
$$

where $S_{r}$ - the amount of subsidence of a point on the surface of the soil at a distance $r$ from the foundation contour; $\left[h_{e}\right]$ - thickness of equivalent soil layer at the point whose subsidence is determined; $P$ - intensity of evenly distributed load.

The thickness of the equivalent soil layer in this case depends on the location of the point relative to the foundation contour and is expressed in general terms by the formula (Tsytovych, 1983):

$$
\left[h_{e}\right]=A \cdot \omega_{c} \cdot b,
$$

where $\omega_{c}$ is the coefficient of the corner point (function $l / b) ; b$ - the width of the corresponding rectangle for which this point is angular.

The coefficient of the corner point $\omega_{c}$ in this case is determined by the formula (Dutchyn \& Melnychenko, 2005):

$$
\omega_{c}(\alpha)=\frac{1}{\pi}[\operatorname{Arch}(\alpha)+\alpha \cdot \operatorname{Arch}(1 / \alpha)],
$$

where $\alpha$ is the aspect ratio of the rectangles of the corner point (in this case $\lim _{\alpha \rightarrow 0} \omega_{c}(\alpha)=0$ ); $\operatorname{Arch}(\alpha)$ is the inverse hyperbolic sine of the quantity $\alpha$, which in the general case is defined as $\operatorname{Arsh}(x)=\ln \left(x+\sqrt{1+x^{2}}\right)$.

Lines of equal soil surface sediments outside the contour of the models of square and rectangular foundations, constructed on the basis of the method of corner points of the equivalent layer using the Surfer software package, are presented respectively in Figures 2 and 3.

According to the results of theoretical studies (Dutchyn \& Melnychenko, 2005), the maximum value of subsidence of the soil surface will be at points located opposite the middle of the long sides of the rectangular foundation (Figure 4).

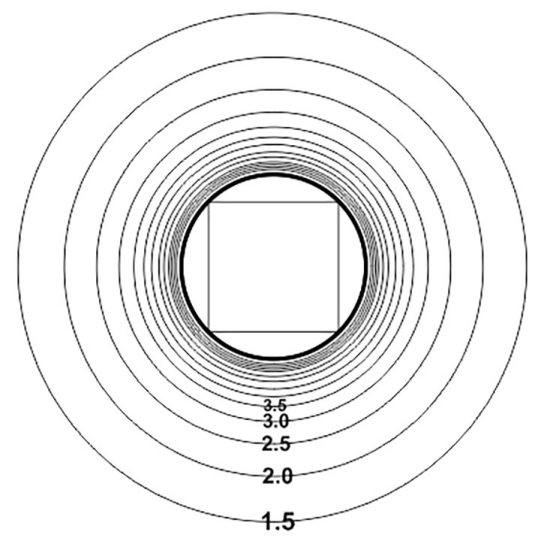

Figure 2. Lines of equal sediments of the soil surface (in $\mathrm{mm}$ ) outside the contour of model No 3 of the square foundation (scale 1: 500)

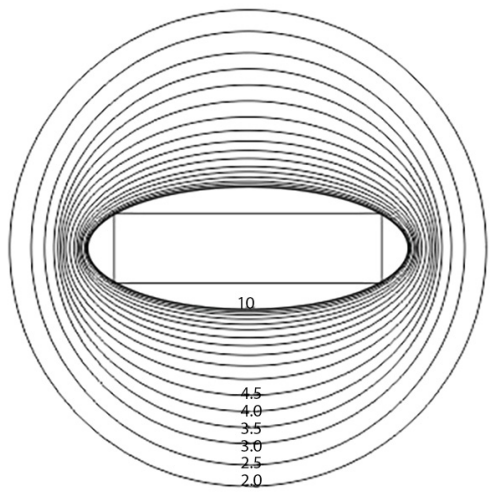

Figure 3. Lines of equal soil surface sediments (in $\mathrm{mm}$ ) outside the contour of model No. 4 of a rectangular foundation (scale 1: 500)

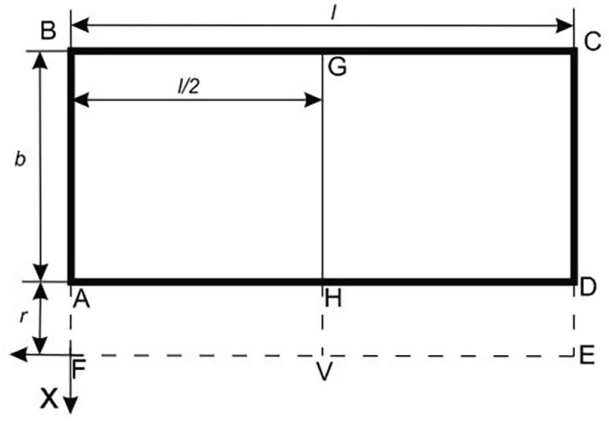

Figure 4. To the determination of the values of the maximum subsidence of the soil surface outside the loaded circuit: $A B C D$ - contour of a rectangular foundation; $r$ - distance from the foundation contour; $V$ - point of the soil surface with a maximum sedimentation value for a given distance

To determine the value of subsidence of the soil surface at the point Vof formula (8) we write in the form (Figure 4):

$$
S_{r_{V}}=\left(h_{e_{V F B G}}+h_{e_{V E C G}}-h_{e_{V F A H}}-h_{e_{V E D H}}\right) m_{v_{m}} P .
$$

According to the results of studies (Dutchyn \& Melnychenko, 2005), the value of subsidence $S_{r}$ of the soil surface outside the foundation contour depends on the value of the average subsidence $S_{m}$ of the foundation, the distance $r$ from the foundation contour and its size $(l, b)$. Therefore, this value can be expressed as a function of the form:

$$
S_{r}=f\left(S_{m}, r, l, b\right) .
$$

The value of the maximum subsidence of the earth's surface outside the loaded circuit can be determined by the formula (Dutchyn \& Melnychenko, 2005):

$$
S_{r}^{\max }=S_{m} \frac{2 a}{\pi}\left(\frac{l}{b}\right)^{\tau} \operatorname{Arsh}[l /(2 r+b)],
$$

where $S_{r}^{\max }$ is the maximum subsidence of the earth's surface at a distance $r$ from the foundation contour; $a$, $\tau$ - coefficients, the values of which are given in formula (6). 
Calculations of maximum subsidence of the soil surface, using the formula (13), were performed on models of rectangular foundations (Table 4).

The calculation results $S_{r}^{\max }$ are shown in Table 4 .

Table 4. Maximum values of subsidence of the soil surface $\left(S_{r}^{\max }\right.$ ) outside the contour of the models of foundations

\begin{tabular}{|c|c|c|c|c|c|}
\hline \multirow{2}{*}{$\begin{array}{c}\text { Distance } \\
r, \mathrm{~m}\end{array}$} & \multicolumn{5}{|c|}{$S_{r}^{\max }, \mathrm{mm}$} \\
\cline { 2 - 6 } & 1 & 2 & 3 & 4 & 5 \\
\hline 5 & 4.59 & 5.70 & 4.78 & 7.69 & 8.53 \\
\hline 10 & 2.85 & 3.76 & 3.13 & 5.11 & 5.31 \\
\hline 20 & 1.59 & 2.19 & 1.84 & 2.99 & 2.99 \\
\hline 30 & 1.10 & 1.53 & 1.50 & 2.10 & 2.07 \\
\hline 40 & 0.84 & 1.17 & 1.01 & 1.62 & 1.58 \\
\hline 50 & 0.68 & 0.95 & 0.82 & 1.31 & 1.28 \\
\hline 60 & 0.57 & 0.79 & 0.69 & 1.10 & 1.07 \\
\hline 70 & 0.49 & 0.68 & 0.60 & 0.95 & 0.93 \\
\hline 80 & 0.43 & 0.60 & 0.53 & 0.84 & 0.81 \\
\hline 90 & 0.39 & 0.54 & 0.47 & 0.75 & 0.73 \\
\hline 100 & 0.35 & 0.48 & 0.43 & 0.67 & 0.65 \\
\hline & & & & & \\
\hline
\end{tabular}

The ratio $S_{r}^{\max } / S_{m}$ taking into account formula (13), we write in the form:

$$
\frac{S_{r}^{\max }}{S_{m}}=\frac{2 a}{\pi}\left(\frac{l}{b}\right)^{\tau} \operatorname{Arsh}[l /(2 r+b)] .
$$

The values of the ratios $S_{r}^{\max } / S_{m}$ are shown in Table 5 .

Table 5. Relationships between soil surface draft ( $\left.S_{r}^{\max }\right)$ and average draft $S_{m}$ of foundation models

\begin{tabular}{|c|c|c|c|c|c|}
\hline \multirow{2}{*}{$\begin{array}{c}\text { Distance } \\
r, \mathrm{~m}\end{array}$} & \multicolumn{5}{|c|}{$\frac{S_{r}^{\max }}{S_{m}}, \%$} \\
\cline { 2 - 6 } & \multicolumn{5}{|c|}{ Model numbers } \\
\cline { 2 - 6 } & 1 & 2 & 3 & 4 & 5 \\
\hline 5 & 33.2 & 40.1 & 27.9 & 41.8 & 33.7 \\
\hline 10 & 20.6 & 26.5 & 18.3 & 27.8 & 21.0 \\
\hline 20 & 11.5 & 15.4 & 10.7 & 16.2 & 11.8 \\
\hline 30 & 8.0 & 10.8 & 8.8 & 11.4 & 8.2 \\
\hline 40 & 6.1 & 8.2 & 5.9 & 8.8 & 6.2 \\
\hline 50 & 4.9 & 6.7 & 4.8 & 7.1 & 5.1 \\
\hline 60 & 4.1 & 5.6 & 4.0 & 6.0 & 4.2 \\
\hline 70 & 3.5 & 4.8 & 3.5 & 5.2 & 3.7 \\
\hline 80 & 3.1 & 4.2 & 3.1 & 4.6 & 3.2 \\
\hline 90 & 2.8 & 3.8 & 2.7 & 4.1 & 2.9 \\
\hline 100 & 2.5 & 3.4 & 2.5 & 3.6 & 2.6 \\
\hline
\end{tabular}

A high-altitude base is a network of reference benchmarks that are installed at some distance from the structure. The subsidence and deformation of the foundations of structures is determined relative to it.

Depending on the nature of the soil and the required accuracy of observations, soil or special depth benchmarks are used.

As is known, the stability of the height position of the benchmark depends largely on the designs of the center, method and depth of its laying.

One of the main factors that determine the instability of geodetic points is the change of loads on the substrate, during which subsidence of the soil surface occurs both under the foundation and beyond.

Depth benchmarks are positioned as close as possible to the structure to minimize height transmission errors. In this case, it is desirable that their anchors be in the indigenous rocks, which are not subject to compression.

The minimum distance to which the depth of reference should be removed from the structure is taken to be equal to half the depth of its laying (Subbotin \& Maznitsky, 1980). The depth of the marking of the points should be lower than the depth of the active zone of compression of the base of the structure. It is believed that such a deepening of the bench will allow taking into account the influence of stresses in the soil caused by the mass of the structure.

According to Shutenko et al. (1989), propagation of stresses in the soil occurs within a cut-off pyramid, the lateral faces of which are located at an angle to the horizon (Figure 5).

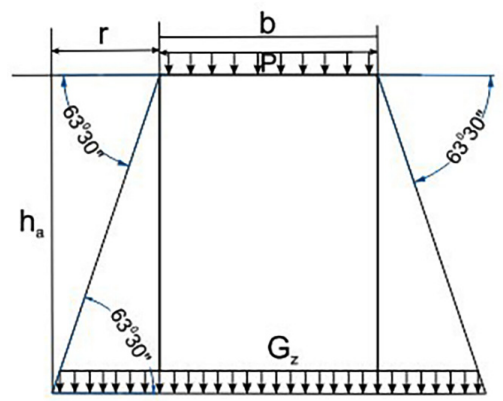

Figure 5. Distribution of additional stresses by the approximate method $63^{\circ} 30^{\prime}$

As can be seen from Figure 5, the stress propagation zone $G_{Z}$ in the plan is a function of the depth of the active compression zone $h_{a}$, i.e.

$$
r=h_{a} \cdot \operatorname{ctg} 63^{\circ} 30^{\prime}=0.5 h_{a} .
$$

Therefore, the minimum distance by which the benchmarks should be removed from the structure is recommended to be taken equal to half the depth of his laying.

But at the same time, as indicated above, a necessary condition is,

$$
h_{\text {benchmarks }} \geq h_{a} ; h_{\text {perr }} \geq h_{a},
$$

where $h_{\text {benchmarks }}$ - the depth of the benchmarks.

In Dalmatov et al. (1986) it is approximately assumed that the spread of the draft sediment to the sides of the 
foundation is more than the size of the active compression zone of the foundation foundation.

The depth of the active compression zone $h_{a}$ in the equivalent soil layer method is defined as

$$
h_{a}=2 h_{e}
$$

where $h_{e}$ is the thickness of the equivalent soil layer.

The values of subsidence of the soil surface at a distance $r=h_{e}$ and $r=h_{a}$ from the foundation contour are shown in Table 6.

Table 6. Probable values of subsidence of the soil surface $S_{r}^{\max }$ at a distance $r=h_{e}$ and $r=h_{a}$ from the contour of the foundation models

\begin{tabular}{|c|c|c|c|c|c|c|}
\hline \multirow{2}{*}{$\begin{array}{c}\text { Model } \\
\text { numbers }\end{array}$} & \multirow{2}{*}{$h_{e}, \mathrm{~m}$} & \multirow{2}{*}{$h_{a}, \mathrm{~m}$} & \multicolumn{2}{|c|}{$S_{r}^{\max }, \mathrm{mm}$} & \multicolumn{2}{c|}{$\frac{S_{r}^{\max }}{S_{m}}, \%$} \\
\cline { 4 - 7 } & & & $r=h_{e}$ & $r=h_{a}$ & $r=h_{e}$ & $r=h_{a}$ \\
\hline 1 & 6.52 & 13.04 & 4.06 & 2.47 & 29.4 & 17.9 \\
\hline 2 & 6.70 & 13.40 & 5.04 & 3.23 & 35.5 & 22.7 \\
\hline 3 & 8.08 & 16.16 & 3.76 & 2.34 & 21.9 & 13.7 \\
\hline 4 & 8.68 & 17.36 & 5.79 & 3.55 & 31.5 & 19.3 \\
\hline 5 & 6.74 & 13.49 & 7.41 & 4.50 & 29.3 & 17.8 \\
\hline
\end{tabular}

As can be seen from Table 6, the subsidence of the soil surface $\left(S_{r}^{\max }\right.$ ) at a distance $r=h_{e}$ (the boundary of the stress zone) from the contour of the rectangular foundation models averages $29.5 \%$, respectively, at a distance $r=$ $h_{a}$ (the likely absorption limit of the subsidence funnel) is $18.3 \%$ of the average subsidence foundation $S_{m}$.

When defining sediments by leveling II and III classes as inirial benchmarks, it is possible to use soil benchmarks, the length of the bench pipe which depends on the depth of freezing ground.

In the first period after laying of soil benchmarks, their settling is possible, which is caused by soil compaction in the pit (structural deformations) (Klimov et al., 1991).

One of the main reasons for the benchmark to settle in the process of structural deformation is its relative weight.

In Bolshakov et al. (1976) for the preliminary calculation of the magnitude of possible subsidence of the benchmark, the formula are used:

$$
S_{\text {benchmarks }}=\sum_{i=1}^{n} P_{o_{i}} \cdot h_{i} \cdot \beta_{3} / E_{o_{i}},
$$

where $P_{o_{i}}$ is the average additional pressure within the $i$-th soil layer; $\beta_{3}$ - transition coefficient, depending on the Poisson's ratio; $E_{o_{i}}$ - modulus of general deformation of the $i$-th soil layer; $h_{i}$ - thickness of the $i$-th soil layer; $n$ - the number of soil layers included in the compression zone.

The transition coefficient $\beta_{3}$ can be expressed through the coefficient of transverse expansion of the soil $\mu_{0}$ (Dashko \& Kachan, 1977):

$$
\beta=1-\frac{2 \mu_{0}^{2}}{1-2 \mu_{0}} .
$$

The modulus of the general soil deformation under the conditions of a one-dimensional problem (where the soil properties do not change significantly along the depth of the core) can be determined by the formula (Tsytovych, 1983):

$$
E_{0}=\frac{\beta}{m_{v}} .
$$

Thus, for the soil benchmarks we write formula (18) in the form:

$$
S_{\text {benchmarks }}=\sum_{i=1}^{n} h \cdot m_{v} \cdot P_{0},
$$

where $h$ is the depth of the center.

The additional pressure $P_{0}$ that arises in the soil after laying the benchmark is determined by the difference between the total pressure from the weight of the point and the pressure from the weight of the soil extracted from the pit (Klimov et al., 1991) that is

$$
P_{0}=P_{\text {benchmarks }}-\gamma \cdot h,
$$

where $\gamma$ is the volumetric weight of the soil.

To determine the conditions under which the calculation of settling centers due to structural deformations is necessary, it is possible to calculate the value of the specific pressure on the soil.

In this case, the weight of the construction of the bench should be calculated so that the pressure created by them on the soil does not exceed the natural, ie.

$$
P_{\text {benchmarks }} \leq \gamma \cdot h .
$$

It was found that soil compaction on the height position of the benchmark does not significantly affect, that is, the sedimentation of the benchmark will be practically zero. Therefore, the subsidence of points of this type can not be calculated.

Another reason that determines the instability of geodetic points is the change in natural pressure due to the loading of the foundations of structures.

Ground benchmarks should be out of the reach of a building or structure. In such cases, it is necessary to take into account the influence of the sedimentation funnel on the displacement of the benchmark, the area of propagation of which, obviously, is not limited to the voltage range.

According to Levchuk et al. (1981), for the construction of industrial and civil construction the distance of soil benchmarks from the structure should not be less than 70-80 m.

There are other tolerances for the removal of soil benchmarks - 50-70 m (Subbotin \& Maznitsky, 1980).

It is likely, that sedimentation of leveling marks, and especially of soil benchmarks, can reproduce sedimentation of the soil surface.

As shown by the results of studies (Uspensky, 1975), the ratio between the magnitudes of vertical movements of the soil surface and changes in the height of soil benchmarks (at the depth of their laying about $1.8 \mathrm{~m}$ ) is in the range of $1.25-1.56$, ie 


$$
S_{\text {benchmarks }} / S_{r}=0.64-0.80 \text {. }
$$

The dependence $S_{\text {benchmarks }} / S_{r}=0.65$ (for the indicated depth) is also confirmed by the results of experimental observations of the vertical movements of the earth's surface and benchmark caused by temperature effects (Pavlik, 1999).

Given the influence of the subsidence zone on the displacement of the soil benchmark and taking the maximum value of the ratio $S_{\text {benchmarks }} / S_{r}=0.80$, formula (24)
is written in the form:

$$
S_{\text {benchmarks }}=0.80 \cdot S_{r}^{\max } \text {. }
$$

Taking into account the dependence (13), the formula (25) will take the form (Dutchyn et al., 2016):

$$
S_{\text {benchmarks }}=0.80 \frac{2 a}{\pi}(l / b)^{f} S_{m} A r s h[l /(2 r+b)] \text {. }
$$

The probable magnitudes of subsidence of soil benchmarks, calculated by the formula (26), depending on the contour of the models of rectangular foundations are shown in Table 7.

Table 7. Probable values of subsidence of soil benchmarks at a distance from the foundation contour

\begin{tabular}{|c|c|c|c|c|c|}
\hline \multirow{2}{*}{$\begin{array}{c}\text { Distance } \\
r, \mathrm{~m}\end{array}$} & \multicolumn{5}{|c|}{$S_{\text {benchmarks }}, \mathrm{mm}$} \\
\cline { 2 - 6 } & 1 & 2 & 3 & 4 & 5 \\
\hline & 3.67 & 4.56 & 3.82 & 6.15 & 6.82 \\
\hline 5 & 2.28 & 3.01 & 2.50 & 4.09 & 4.25 \\
\hline 10 & 1.75 & 1.47 & 2.39 & 2.39 \\
\hline 20 & 1.27 & 1.75 & 1.66 \\
\hline 30 & 0.88 & 1.22 & 1.20 & 1.68 & 1.26 \\
\hline 40 & 0.67 & 0.94 & 0.81 & 1.30 & 1.26 \\
\hline 50 & 0.54 & 0.76 & 0.66 & 1.05 & 1.02 \\
\hline 60 & 0.46 & 0.63 & 0.55 & 0.88 & 0.86 \\
\hline 70 & 0.39 & 0.54 & 0.48 & 0.76 & 0.74 \\
\hline 80 & 0.34 & 0.48 & 0.42 & 0.67 & 0.65 \\
\hline 90 & 0.31 & 0.43 & 0.38 & 0.60 & 0.58 \\
\hline 100 & 0.28 & 0.38 & 0.34 & 0.54 & 0.52 \\
\hline
\end{tabular}

The probable magnitudes of subsidence of soil benchmarks at a distance (the probable limit of propagation of the subsidence funnel) from the contour of the foundation models are shown in Table 8.

As can be seen from Table 8 , the subsidence of soil benchmarks at a distance $r=h_{a}$ from the contour of rectangular foundations averages $14.6 \%$ of the average subsidence $S_{m}$ of the foundation.

As a rule, soil benchmarks are not installed at such a distance $\left(r=h_{a}\right)$ from the foundation.

Depending on the importance of the observation results, the corresponding ratio between the error and the
Table 8. The probable values of of subsidence of soil benchmarks $S_{\text {benchmarks }}$ at a distance from the contour of the models of rectangular foundations

\begin{tabular}{|c|c|c|c|}
\hline Model numbers & $\begin{array}{c}h_{a}, \\
\mathrm{~m}\end{array}$ & $\begin{array}{c}S_{\text {benchmarks }}, \\
\mathrm{mm}\end{array}$ & $\begin{array}{c}S_{\text {benchmarks }} / S_{m}, \\
\%\end{array}$ \\
\hline 1 & 13.04 & 1.98 & 14.3 \\
\hline 2 & 13.40 & 2.58 & 18.2 \\
\hline 3 & 16.16 & 1.87 & 10.9 \\
\hline 4 & 17.36 & 2.84 & 15.4 \\
\hline 5 & 13.49 & 3.60 & 14.2 \\
\hline
\end{tabular}

expected result of the measurement of deformation (draft) is also chosen.

From importance of the results of observations, the appropriate ratio between the error and the expected result of the measurement of deformation (subsidence) is choosing.

If the marginal error in the determination of subsidence $S_{m}$ is denoted by $\Delta S_{m}$, and the reliability coefficient later $k$, then

$$
\Delta S_{m}=k \cdot S_{m} .
$$

Given the requirements for the accuracy of determining parameters in construction (Lukyanov, 1981), and taking the reliability coefficient $k=0.10$ (for especially important works), we write the formula (27) in the form:

$$
\Delta S_{m}=0.10 \cdot S_{m} .
$$

The total expected marginal error in determining subsidence $\Delta S_{m}$, as is known, is calculated by the formula:

$$
\Delta S_{m=} \sqrt{\Delta_{\text {benchmarks }}^{2}+\Delta_{\text {lok }}^{2}},
$$

where $\Delta_{\text {benchmarks }}$ - the marginal error of determining the subsidence of the initial benchmark for the local leveling network; $\Delta_{l o k}-$ marginal error in determining the subsidence of the estimated point from the initial benchmark, taking into account all local network's connections.

Assessment of the stability of the initial benchmarks, in the general case, consists of dividing the obtained data by the offset and on the possible errors in their determination.

If the amount of sedimentation does not exceed the margin of error of its definition, then the benchmark is considered stable, and vice versa.

For the marginal error of determining the subsidence of the initial benchmark, we take $1 / 2$ the marginal error of determining the subsidence value of the foundation of the structure,

$$
\Delta_{\text {benchmarks }}=0.5 \Delta S_{m} .
$$

In this case, the condition of stability of soil benchmarks, taking into account formulas (28) and (30), we write in the form:

$$
S_{\text {benchmarks }} \leq 0.05 S_{m} .
$$


The marginal error in determining the subsidence of the estimated point of the structure from the original benchmark of formula (29) will be

$$
\Delta_{\text {lok }}=\sqrt{\Delta S_{m}^{2}+\Delta_{\text {benchmarks }}^{2}} .
$$

The results of the estimation of the accuracy of determining the subsidence of foundations and soil benchmarks by formulas (28), (30), (32) are shown in Table 9.

Table 9. Evaluation of the accuracy of determining the average sediment models of foundations and soil benchmarks

\begin{tabular}{|c|c|c|c|c|}
\hline $\begin{array}{c}\text { Model } \\
\text { numbers }\end{array}$ & $\begin{array}{c}S_{m}, \\
\mathrm{~mm}\end{array}$ & $\begin{array}{c}\Delta S_{m}, \\
\mathrm{~mm}\end{array}$ & $\begin{array}{c}\Delta_{\text {benchmarks }}, \\
\mathrm{mm}\end{array}$ & $\begin{array}{c}\Delta_{\text {lok }}, \\
\mathrm{mm}\end{array}$ \\
\hline 1 & 13.82 & 1.38 & 0.69 & 1.20 \\
\hline 2 & 14.21 & 1.42 & 0.71 & 1.23 \\
\hline 3 & 17.13 & 1.71 & 0.86 & 1.48 \\
\hline 4 & 18.40 & 1.84 & 0.92 & 1.59 \\
\hline 5 & 25.30 & 2.53 & 1.26 & 2.19 \\
\hline
\end{tabular}

The ratio between the sediments of the soil benchmarks and the foundation of the structure are shown in Table 10.

Table 10. The values of the ratios between the sediments of the soil benchmarks ( $\left.S_{\text {benchmarks }}\right)$ and the foundation of the structure $\left(S_{m}\right)$

\begin{tabular}{|c|c|c|c|c|c|}
\hline \multirow{2}{*}{$\begin{array}{c}\text { Distance } \\
r, \mathrm{~m}\end{array}$} & \multicolumn{5}{|c|}{$S_{\text {benchmarks }} / S_{m}, \%$} \\
\cline { 2 - 6 } & 1 & 2 & 3 & 4 & 5 \\
\hline & \multicolumn{5}{|c|}{ Model numbers } \\
\hline 5 & 26.6 & 32.1 & 22.3 & 33.4 & 27.0 \\
\hline 10 & 16.5 & 21.2 & 14.6 & 22.2 & 16.8 \\
\hline 20 & 9.2 & 13.3 & 8.6 & 13.0 & 9.4 \\
\hline 30 & 6.4 & 8.6 & 7.0 & 9.1 & 6.6 \\
\hline 40 & 4.9 & 6.6 & 4.7 & 7.0 & 5.0 \\
\hline 50 & 3.9 & 5.3 & 3.8 & 5.7 & 4.0 \\
\hline 60 & 3.3 & 4.4 & 3.2 & 4.8 & 3.4 \\
\hline 70 & 2.8 & 3.8 & 2.8 & 4.1 & 2.9 \\
\hline 80 & 2.5 & 3.4 & 2.5 & 3.6 & 2.6 \\
\hline 90 & 2.3 & 3.0 & 2.2 & 3.3 & 2.3 \\
\hline 100 & 2.0 & 2.7 & 2.0 & 2.9 & 2.1 \\
\hline
\end{tabular}

In accordance with formula (31), to ensure the stability of the benchmarks, the ratios $S_{\text {benchmarks }} / S_{m}$ should not
exceed $5 \%$,

$$
S_{\text {benchmarks }} / S_{m}<0.05 \text {. }
$$

In turn, the permissible value of the ratio between the subsidence of the soil surface and the foundation of the structure to ensure the necessary stability of the original benchmarks will be determined on the basis of formulas (25) and (31):

$$
S_{r}^{\max } / S_{m}<0.0625
$$

Thus, according to the calculations presented on the models of flexible foundations (Tables 5,10 ), the probable minimum distance $r^{\mathrm{min}}$ ) from the foundation contour to the laying places of the output soil frames should not exceed the values given in Table 11 .

Table 11. Estimated minimum distance from the foundation contour to the laying places of the output soil benchmarks

\begin{tabular}{|c|c|c|c|c|c|}
\hline Model numbers & 1 & 2 & 3 & 4 & 5 \\
\hline$r^{\min }, \mathrm{m}$ & 40 & 60 & 40 & 60 & 50 \\
\hline
\end{tabular}

The calculations were carried out on a separate foundation without taking into account the possible imposition of force fields in the soil array from the loading. Other criteria may be adopted to evaluate the accuracy of determining the settlements of the foundations of engineering structures and local level points.

\section{Conclusions}

1. It is established that the values of settlements of the surface of the soil, calculated by the method of angular points of the equivalent layer on models of rectangular foundations, at a distance equal to the depth of the active compression zone (the probable limit of propagation of the funnel of sedimentation) are on average $18 \%$ of the value of the average subsidence of the foundation.

2. In order to evaluate the effect of static loads of the foundations of the structures on the subsidence of the soil surface, the lines of equal settlements of the soil surface outside the contour of the foundations of square and rectangular shapes were constructed.

3. To ensure the stability of soil benchmarks taken over the initial, the subsidence of the soil surface shall not exceed $6 \%$ of the average subsidence of the foundation of the structure, and the subsidence of the soil benchmark $\sim 5 \%$ of this value.

Given the above ratios, the probable optimum distance from the contour of the foundation of the structure to the places of laying of the original soil benchmarks will be determined.

\section{Disclosure statement}

The authors declare no conflict of interest.

\section{References}

Bolshakov, V. D., Vasyutinsky, I. Yu., Klyushin, E. B., Lebedev, N. N., Novak, V. E., Piskunov, M. E., Ryazantsev, G. E., \& Yambaev, Kh. K. (1976). Methods and instruments for highprecision geodetic measurements in construction. Nedra.

Ganshin, V. N., Storozhenko, A. F., \& Ilyin, A. G. (1981). Measurement of vertical offset structures and stability analysis of benchmarks. Nedra. 
Dashko, G. D., \& Kachan, A. A. (1977). Soil mechanics in engineering-geological practice. Nedra.

Dalmatov, B. I., Morareskul, N. N., \& Naumenko, V. G. (1986). Designing the foundations of buildings and industrial structures. Higher school.

Dutchyn, M. M. (1999). By sizing, the spread of the settling funnel is influenced by the static loads of the foundations of structures. Modern Achievements of Geodesy, Geodynamics and Geodesic Production, 1(1), 80-82.

Dutchyn, M. M. (1996). The study of sediment foundations of gas pumping units of compressor stations based on the physical and mechanical properties of soils. Geodesy, Cartography and Aerial Photography, 57, 16-26.

Dutchyn, M. M. (2001). To determine the accuracy of the sediment foundations of gas pumping units of compressor stations, depending on the location of the deformation grades. Geodesy Engineering, 45, 73-76.

Dutchyn, M. M., \& Melnychenko, G. G. (2005). To determine the maximum subsidence of the soil surface outside the loaded contour. Modern Achievements of Geodetic Science and Production, I, 160-163.

Dutchyn, M. M. (2005). Investigation of the accuracy and information content of the determination of sediment foundations of engineering structures. Modern Achievements of Geodetic Science and Production, 1(9), 164-168.

Dutchyn, M. M., Melnychenko, G. G., \& Bida, I. V. (2007). Determining the optimal position of deformation grades when observing the vertical movements of the foundations of gas compressor station structures. Modern Achievements of Geodetic Science and Production, 14, 95-100.

Dutchyn, M. M., Ilkiv, E. U., \& Bida, I. V. (2012a, October 2427). Investigation of soil surface sediment outside the loaded contour. In New technologies in geodesy, land management and nature management: coll. materials of VI International Science-Pract. Conf. (pp. 68-70). Uzhgorod, Ukraine.

Dutchyn, M. M., Melnychenko, G. G., Bida, I. V., \& Skryl, V. A. (2012b). Investigation of the deformation characteristics of the foundations of gas pumping units of compressor stations. Modern Achievements of Geodetic Science and Production, 2(24), 86-88.

Dutchyn, M. M., Grytsyuk, T. Yu., \& Nychvyd, M. P. (2014a). Investigation of the accuracy of determining the subsidence of foundations of engineering structures at the stage of soil compaction. Modern Achievements of Geodetic Science and Production, 27, 55-58.

Dutchyn, M. M., \& Melnychenko, G. G. (2002). To the determination of allowable drawdown values of gas pumping units of compressor stations. Modern Achievements of Geodetic Science and Production, 1(3), 190-192.
Dutchyn, M. M., Ilkiv, E. Yu., \& Galyarnik, M. V. (2014b, November 5-6). Features of the use of soil benchmarks when observing subsidence foundations of engineering structures. In Geodesy. Land tenure. Nature Management: coll. materials of All-Ukrainian scientific-practical conference (pp. 17-19). Rivne, Ukraine.

Dutchyn, M. M., Grytsyuk, T. Yu., \& Bida, I. V. (2016, October $6-8)$. The study of informativeness of the sediment of the soil surface and rappers under the influence of static loads. In New technologies in geodesy, land management and nature management: coll. materials of VIII International SciencePract. Conf. (pp. 99-103). Uzhgorod, Ukraine.

Klimov, A. D., Kalugin, V. V., \& Pisarenko, V. K. (1991). Workshop on applied geodesy. Surveys, design and construction of engineering structures. Nedra.

Levchuk, G. P., Novak, V. E., Bolshakov, V. D., Lebedev, N. N., Bakanova, V. V., Glotov, G. F., Markuze, Yu. I., Piskunov, M. E., Zatsarinny, V. V., Bronshtein, G. S., Zaitsev, A. K., Klyushin, E. B., Klimov, O. D., Yambaev, Kh. K., Barkov, D. P., Mikhelev, D. Sh., Marfenko, S. V., Gorbenko, O. I., Vasyutinsky, I. Yu., \& Bush, V. V. (1980). Reference guide for engineering and geodetic works. Nedra.

Levchuk, G. P., Novak, V. E., \& Konusov, V. G. (1981). Basic methods and principles of engineering and geodetic works. Nedra.

Lukyanov, V. F. (1981). Calculations of the accuracy of engineering and geodetic works. Nedra.

Pavlik, V. G. (1999). Comprehensive study of seasonal hydrothermal deformations of the earth's surface: [Doctoral Dissertation]. Lviv, Ukraine.

Perovich, L. M., \& Dutchyn, M. M. (1996). Monitoring of deformations of engineering structures at gas transport facilities. In Geoinformational environmental monitoring (pp. 90-91).

Rydy, G. M., Dutchyn, M. M., \& Melnychenko, G. G. (2001). The calculation of the sediment foundations of gas compressor stations by the method of corner points. Exploration and Development of Oil and Gas Fields, 38, 151-153.

Shutenko, A. N., Gilman, D. D., \& Lupan, Yu. G. (1989). Soil mechanics. Higher Shk.

Subbotin, I. E., \& Maznitsky, A. (1980). Handbook of a builder in engineering geodesy. Budivelnyk.

Tishin, V. G. (1985). Foundations and foundations of oil and gas industry objects. Nedra.

Tsytovych, N. A. (1983). Soil mechanics. Higher Shk.

Uspensky, M. (1975). On the study of the influence of technogenic processes on the deformation of the earth's surface and the stability of geodetic points. Geodesy and Cartography, 4, 8-11.

Zurnadzhi, V. A., \& Nikolaev, V. V. (1967). Soil mechanics, foundations and foundations. Higher Shk. 